\title{
Unfolding Participation. \\ What do we mean by participation - conceptually and in practice
}

\author{
Joanna Saad-Sulonen \\ Centre for Participatory IT \\ Aarhus University, Denmark \\ +4587156212 \\ jss@cs.au.dk \\ John Vines \\ Digital Interaction Group \\ Culture Lab \\ Newcastle University, UK \\ john.vines@newcastle.ac.uk
}

\author{
Kim Halskov \\ Centre for Participatory IT \\ Aarhus University, Denmark \\ +4587161982 \\ halskov@cavi.au.dk \\ Eva Eriksson \\ Department of Applied IT \\ Chalmers University of \\ Technology, Sweden \\ eva.eriksson@chalmers.se
}

\author{
Liesbeth Huybrechts \\ Faculty of Architecture and Art \\ University of Hasselt, Belgium \\ +32486158840 \\ liesbeth.huybrechts@uhasselt.be \\ Helena Karasti \\ Information Systems \\ Luleå University of Technology \\ Luleå, Sweden \\ helena.karasti@1tu.se
}

\begin{abstract}
The aim of the Unfolding Participation workshop is to outline an agenda for the next 10 years of participatory design (PD) and participatory human computer interaction (HCI) research. We will do that through a double strategy: 1) by critically interrogating the concept of participation (unfolding the concept itself), while at the same time, 2) reflecting on the way that participation unfolds across different participatory configurations. We invite researchers and practitioners from PD and HCI and fields in which information technology mediated participation is embedded (e.g. in political studies, urban planning, participatory arts, business, science and technology studies) to bring a plurality of perspectives and expertise related to participation.
\end{abstract}

\section{Author Keywords}

Participation; participatory design; design events; information technology; IT-mediated participation; democracy

\section{ACM Classification Keywords}

H5 [Information interfaces and presentation]: User interfaces - user-centered design.

\section{DESCRIPTION OF THE WORKSHOP}

The last few years have witnessed attempts from various research communities interested in participatory approaches to information technology (IT) to define what they mean by

Copyright $\subset 2015$ is held by the author(s). Publication rights licensed to Aarhus University and ACM

5th Decennial Aarhus Conference on Critical Alternatives

August 17 -21, 2015, Aarhus Denmark

DOI: http://dx.doi.org/10.7146/aahcc.v1i1.21471 participation. Whereas much of the conceptual and ideological grounding for participation in IT comes from the field of Participatory Design (PD), even there, the definitions of participation are varied and often not explicitly articulated [2]. A recent report by $\mathrm{CHI}$ community members also highlights the need for mapping the various definitions of the term participation used in the field as well as identifying the ways these definitions are shaped by interaction with other discourses [6]. Clearly, it is time for us as a community of participatory designers and researchers to question what we exactly mean when we say "participation" and how it is reflected in our practice.

PD has always been motivated by a concern for improving the quality of life and work situations by designing critical alternatives with a more or less pronounced utopian agenda. These designed alternatives come in the form of products, coupled with an engagement in politics that was especially key in shaping the original Scandinavian PD approach. Participatory design takes place by involving people in the design process, in increasingly varying contexts. Participatory methods are deployed during participatory design events staged by designers in order to make this involvement possible. (See Figure 1;[2]).

Participation in PD research has had a strong focus on exploratory design projects and so far has often taken place through single design events (e.g. an ethnographic field study or a workshop), where issues such as 'involving users', including the users point of view, and participation as a matter of mutual learning have been in the foreground. 


\begin{tabular}{|l|l|}
\hline Politics & $\begin{array}{l}\text { People who are affected by a decision should have an } \\
\text { opportunity to influence it. }\end{array}$ \\
\hline People & $\begin{array}{l}\text { People play critical roles in design by being experts in their } \\
\text { own lives. }\end{array}$ \\
\hline Context & $\begin{array}{l}\text { The use situation is the fundamental starting point for the } \\
\text { design process. }\end{array}$ \\
\hline Methods & $\begin{array}{l}\text { Methods are means for users to gain influence in design } \\
\text { processes. }\end{array}$ \\
\hline Product & $\begin{array}{l}\text { The goal of participation is to design alternatives, improving } \\
\text { quality of life. }\end{array}$ \\
\hline
\end{tabular}

Figure 1: The five fundamental aspects of PD

Today, we witness many different other types of participations that also combine IT and politics. For example, participation related to decision-making, dialogue and debate around matters of concern are increasingly occurring online, mediated by IT during use time. It happens at the grassroots level through participatory IT (Web 2.0), or is set up by authorities or other actors engaged in triggering participation. If this current trend continues, what will be the role of the staged participatory design event, which is based on in-person engagement with others? What kind of new participatory configurations can emerge? In what ways can they support the original values of PD around democracy, quality of working life and politics?

The aim of the Unfolding Participation workshop is to outline an agenda for the next 10 years of participatory design and participatory $\mathrm{HCI}$ research. We will do that through a double strategy: 1) by critically interrogating the concept of participation (unfolding the concept itself), while at the same time 2) reflecting on the way that participation unfolds across different participatory configurations. We will build upon the discussion started by [2] and [6] and ask: What counts as participation in IT-related fields? How does participation unfold over time and across different participatory configurations? These questions are timely and open up a space for challenging the way participation often takes place in the fields of PD and participatory HCI. Initiating this discussion would benefit from the face-toface type of interaction and debate that a workshop format brings. We invite researchers and practitioners from PD and $\mathrm{HCI}$ and fields in which IT-mediated participation is embedded (e.g. in political studies, urban planning, participatory arts, business, science and technology studies) to reflect together on: i) how participation has unfolded in projects or case studies in which they have been involved (during and in-between design events), ii) how has it connected to types of participations other than design events, and iii) how does their own participatory practice relate to IT-mediated participation and participatory IT specifically?

Contributions to the workshop may expand one or more of the following key issues:
- How can we map, analyse and reflect on how participation is unfolding over time and in different configurations?

- How does participation during and in-between design events link to other forms of participation, such as what is increasingly being referred to as infrastructuring [4]?

- What do participatory IT and IT mediated participation mean during and in-between design events?

- What are current democratic drivers for participation?

- What are possible combinations of different types of participation (design of IT + others) [5]?

- When we talk about participation, do we mean the same thing in different fields [3]? How to engage in exchange and collaboration with other fields that also deal with participation, but also with all those involved in practice?

- What are the challenges for participation in largescale configurations [1]?

The workshop will cover one full day (09:00-16:30 on $17^{\text {th }}$ of August 2015). The optimal number of participants would be approximately 15 . Groups will be pre-formed by the organisers around themes identified in the position papers. The planned activities for the day are as follows: 1) introduction to the day's programme by the organisers and division of the participants in groups; 2) quick pitches by the participants in their group and group discussions around the key topics; 3) identifying key challenges through group work; 4) from challenges to sketching an agenda for the next 10 years (collaborative ideation session resulting in a DIY poster to be displayed during the conference); 5) planning for publishing a join workshop summary report and other possible further publications.

The workshop organizers all share an interest in participation but approach it from different angles:

Kim Halskov is professor in interaction design at The Department of Aesthetics and Communication at Aarhus University, Denmark where he in addition to being director of Centre for Advanced Visualization and Interaction, see CAVI.au.dk, also is co-director of the Centre for Participatory IT, see PIT.au.dk. From a background in participatory design Kim Halskov's research areas includes innovation processes, design processes, and experience design.

Joanna Saad-Sulonen is a postdoctoral researcher at the Centre for Participatory IT at Aarhus University. She has a background in architecture, new media and digital design. She recently defended her $\mathrm{PhD}$ on the topic of Combining Participations where she claims that participatory approaches to the design of IT can be combined with participatory approaches in urban planning. 
Eva Eriksson is a lecturer in Interaction design at the Department of Applied IT, Chalmers University of Technology, in Gothenburg, Sweden, where she is also responsible for the IXDCTH design labs. She has experience from large-scale participation, $\mathrm{PD}$ in digital fabrication in schools, and is one of the founders of IDAC Gothenburg Working Group for Interaction Design and Children (IDAC.se) - a research group focused on designing interaction for and with children.

Liesbeth Huybrechts is a Postdoctoral researcher in the area of participatory design, media, architecture and planning in the research group Arck (University of Hasselt). Moreover, she is involved in the masters Social Design in Design Academy of Eindhoven. She was co-founder of the research group Social Spaces (KULeuven), exploring the social meanings, uses and applications of design and art. She is currently involved in the research project Traders (FP7, Marie Curie), dealing with the exploration of definitions and practices of Participatory Design and Art in Public Space (www.tr-aders.eu) and WeforWork Genk, researching contemporary workspaces and forms in participatory ways.

John Vines is Lecturer in Interaction Design Methods, based within the Digital Interaction Group at Culture Lab, Newcastle University. His research concerns the study and development of methods and techniques for involving people in design processes, and has a particular interest in the role of digital technology in supporting wider citizen engagement in matters of local concern, including neighborhood services and planning, social care and civic engagement. He also has ongoing research exploring what the term 'participation' means in the field of HCI, especially in a period where boundaries between disciplines engaging in participatory activity are increasingly blurred.

Helena Karasti, professor in information systems at Luleå University of Technology, Sweden, has a long-term research interest in the integrations of participatory design and ethnography. She has studied participatory design in settings where no professional designers were involved, thus giving rise to different understandings of participation(s). Driven with interest in ethnography and critical research traditions she has addressed the notions and practices of participation from the vantage point of reflexivity. She has contributed to research on infrastructuring where the issues of participation are revisited due to the complexities and extended scopes involved.

\section{CALL FOR PARTICIPATION}

The aim of the Unfolding Participation workshop is to outline an agenda for the next 10 years of participatory design and participatory HCI research. We will do that through a double strategy: 1) by critically interrogating the concept of participation (unfolding the concept itself), while at the same time, 2) by reflecting on the way that participation unfolds across different participatory configurations. We will continue the discussion started by [2] and [6] related to the current fuzziness in defining participation in participatory design and participatory HCI research, and ask: What counts as participation in IT-related fields? How does participation unfold over time and across different participatory configurations?

PD research has a strong history in participation for democratic goals and has brought forward the need to consider these goals while at the same time opening up participation in the design of IT. So far, participation in PD research has often taken place through exploratory design projects and single design events (e.g. an ethnographic field study or a workshop), where issues such as 'involving users', including the users point of view, and participation as a matter of mutual learning have been in the foreground. Today, we witness many different other types of participations that also combine IT and politics, but not in the traditional PD manner. For example, participation related to decision-making, dialogue and debate around matters of concern are increasingly occurring online, mediated by IT during use time. What does this mean for PD? There is a pressing need to explore how staged participation unfolds over time and design events and how it might link to other forms of participation.

The workshop will kick start joint reflection on shaping a research agenda around participation for the next ten years by identifying challenges that $\mathrm{PD}$ and participatory $\mathrm{HCI}$ would need to debate. Given that the issues invoked here cross many disciplinary boundaries, we invite submissions from researchers and practitioners from a myriad of fields and disciplines to bring a plurality of perspectives and expertise related to participation. We therefore invite work from not just those situated in participatory design and human-computer interaction but also from fields such as political studies, urban planning, participatory arts, business, and science and technology studies, among others. Potential participants are asked to reflect on the following: i) How has participation unfolded in projects or case studies in which they have been involved (during and in-between design events)? ii) How has it connected to types of participations other than design events? iii) How does their own participatory practice relate to IT-mediated participation and participatory IT specifically?

The workshop aims to bring forward the need for a contemporary take on participation and how it relates to democracy, and IT. Where do these concerns stand now when focus has for a while been more on the staging of participation through design events, but also when IT is increasingly mediating other forms of participation?

The key issues to address include (but are not limited to):

- How can we map, analyse and reflect on how participation is unfolding over time and participatory configurations? 
- How does participation during and in-between design events link to other forms of participation, such as what is increasingly being referred to as infrastructuring?

- What do participatory IT and IT mediated participation mean during and in-between design events?

- What are current democratic drivers for participation?

- What are possible combinations of different types of participation (design of IT + others)?

- When we talk about participation, do we mean the same thing in different fields? How to engage in exchange and collaboration with other fields that also deal with participation, but also with all those involved in practice?

- What are the challenges for participation in largescale configurations?

The above mentioned key issues can be addressed and expanded by reflecting on these more general challenges related to participation and how they unfold in the particular context of participation with and for IT:

- The shaping and unfolding of the participatory processes over time: What are the conditions that shape participation? What are the dynamics of participations? How and when can participation be sustained, and why? What is the role of IT?

- The boundaries of participation: When does participation begin and end?

- Scandinavia is the historical home of PD, but also the place where a certain type of democracy has been and is being practiced. How does this shape our practice of participation? What does it mean when we are at the same time part of a wider global reality where different modes of production and sharing are shaping new cultures of participation?

We invite submissions of positions papers as 2-4 pages PDF documents following the SIGCHI publications template (http://www.sigchi.org/publications/chipubform). Submissions need to address the main topic of the workshop as well as one or more of the key issues. The optimal number of participants is around 15. Selection will be based on the relevance of the position papers with regards to the theme and key issues of the workshop. The selected position papers will be published on the workshop website: http://pit.au.dk/unfoldingparticipation. The main outcomes of the workshop will be summarized into a coauthored joint workshop report/publication.

\section{RECRUITMENT STRATEGY}

The call for participation will be distributed from mid March to mid May 2015. The workshop coordinators will use their connections with key research centers in HCI, design, architecture, urban planning, and science and technology studies, to distribute the call for participation. They will be able to reach significant PD, participatory $\mathrm{HCI}$, as well participatory planning audiences worldwide. The call will also be distributed through key mailing lists including CHI announcement, NordiCHI announcement, Design Research Society and PD mailing lists. We will also be using social media as a means for engaging with potential workshop participants, supplemented by a frequently updated workshop website hosted on the Aarhus University servers.

All submissions will be peer reviewed by the workshop organizers. The review and selection process will aim to ensure that the papers address the theme and key issues outlines in the call for papers: Does the paper address the theme of participation in (or with) IT? Does the paper provide critical reflection on what counts as participation? Does the paper address one or more of the following key themes: 1) The unfolding of participation over time and participatory configurations 2) Different types of participations: design events, infrastructuring, IT-mediated participation and possible relationships between them 3) Contemporary democratic drivers of participation 4) The need for a coming of age of research on participation and the need for trans-disciplinary approaches

We will inform authors of decisions and of any requested changes to their papers approximately three weeks after the submission deadline. We will use the workshop website, along with email, as a way of frequently updating accepted participants. The participants will be given the opportunity to read each other's work before the workshop.

\section{REFERENCES}

[1] Dalsgaard P., Eriksson E. Large-Scale participation: A case study of a Participatory Approach to Developing a New Public Library. In proceedings of CHI. Paris, France, (2013). ACM press, New York.

[2] Halskov, K. \& Hansen, N.B. The diversity of participatory design research practice at PDC 20022012. In International Journal of Human-Computer Studies. (2014).

[3] Huybrechts, L. (ed.) Participation is Risky. Approaches to Joint Creative Processes. Amsterdam: Valiz. 2014.

[4] Karasti, H. Infrastructuring in participatory design. In Proc. of PDC'14. (2014). ACM Press

[5] Saad-Sulonen, J. Combining Participations. Doctoral dissertation. Helsinki: Aalto University. 2014.

[6] Vines, J., Clarke, R., Wright, P., McCarthy, J., Olivier, P. Summary Report on CHI 2012 invited SIG: Participation and HCI: Why Involve People in Design? (2012). 